\title{
Ne quid nimis [1]
}

Vier Jahre Tätigkeit im Zentralvorstand zum Thema Prävention und Gesundheitsförderung führen mich zu einer vorausblickenden Hinterfragung der Tendenzen auf diesem Gebiet.

Prävention und Gesundheitsförderung werden in der Gesundheitspolitik aller europäischen Länder immer wichtiger. Die politischen Stossrichtungen sind vielfältig und reichen von der halbherzigen Untersuchung der Auswirkungen der Lebensmittelindustrie auf die Gesundheit (Zucker, Fett, Salz etc.) über die Motorenindustrie (Feinstaub) und den Städtebau (Lärm, Luft etc.) bis zur zahlengestützten Jagd auf ein als «Risiko» bezeichnetes Einzelverhalten.

«Der Mensch muss vor sich selbst geschützt werden!», «Die Allgemeinheit hat nicht für das schädliche Verhalten verantwortungsloser Einzelner zu bezahlen!»: Die Slogans sind bekannt. Und deshalb muss reglementiert und legiferiert werden. Tabak, Ernährung, Geschwindigkeit, Sex, Suchtmittel: alles wird unter dem Gesichtspunkt des sogenannten «Risikoverhaltens» behandelt. Aber «wie weit muss der Staat die Menschen vor ihren eigenen Lastern sschützen?? Wann kann man sagen, dass das Verhalten von Einzelnen oder «Minderheiten> der Allgemeinheit schadet?» [2]. Es gibt tatsächlich einen Trend zum Zuviel als leuchtendes Symptom unserer modernen Welt. Indem man um jeden Preis (aber auch zu einem günstigen Preis) das Gute will, führt man letztlich eine Logik des Schlechtesten ein, deren exorbitante Kosten nur teilweise durch die Anwendung eines Gesetzes, einer ISO-Norm oder eines von einem Expertengremium abgesegneten Protokolls gedeckt werden. In dieser wohl bald als «Kollektivwahn» zu bezeichnenden Situation versuchen vom Klinikalltag abgekoppelte Wissenschaftler und Gesundheitsökonomen die verhaltensorientierten und mikroökonomischen Indikatoren des Wohlbefindens zu identifizieren [3]. Hierbei wird jedoch Wohlbefinden mit Gesundheit verwechselt. Die Idee von Solidarität in der Grundversicherung sagt nichts über eine politische linke oder rechte - Ideologie aus. Es handelt sich vielmehr um eine Reaktion auf die konkrete Tatsache der biogenetischen Ungleichheit der Menschen. Und diese wird weder durch eine prädiktive Medizin noch eine Verhaltensreglementierung verringert.

Wie sieht der positive Gedanke auf dem Gebiet der Prävention und Gesundheitsförderung aus? Es ist ein Gedanke, der sich als gerecht und pragmatisch versteht, ein Gedanke auf der Basis von Messung und Bewertung der Kosten eines Verhaltens, ein Gedanke, der folglich Normen und Vorgaben für das richtige Verhalten festlegt, aber nicht zum Wohl des Einzelnen, sondern zugunsten der wirtschaftlichen Logik des Immer-Mehr. Vielleicht sollte daran erinnert werden, dass nur der einzelne Mensch selbst die subjektiven Merkmale seiner Gesundheit und seines Wohlbefindens festlegen und eruieren kann. Der positive Gedanke, den man uns präsentiert, ist voluntaristisch, interventionistisch und entsubjektivierend - einige würden sagen «neopaternalistisch».

Dies bedeutet jedoch nicht, dass ich gegen Prävention und Gesundheitsförderung bin. Ich hinterfrage lediglich die Anwendungsmodalitäten und den Platz, den die Ärzteschaft in dieser Entwicklung einnehmen wird. Ehrlich gesagt weiss ich nicht, ob diese voluntaristische und prädiktive Politik noch praktizierende Ärzte brauchen wird. Diese neopaternalistische Politik, die sich an den Bürger wendet, umgeht zunehmend den Arzt als Garanten einer privaten, einzigartigen und auf eine fallweise Beurteilung gestützten therapeutischen Beziehung. Sie braucht das Einzigartige nicht, weil sie auf dem positivistischen Gedanken des «Allgemeinwohls» basiert, der um seine Ziele zu erreichen - die kognitive und verhaltensorientierte Logik missbraucht, deren Merkmale Gesetze, Verbote, Strafen und schliesslich Diskriminierung sind. Vergessen geht dabei allerdings die unwiderlegbare Feststellung, dass es nicht reicht, das «richtige Verhalten» gestützt auf Zahlen einer klinischen Wirklichkeit aufzuzwingen oder zu predigen, damit der Betroffene begreift, was dies subjektiv bedeutet. Der Arzt, der dies vergisst, verliert die Fähigkeit, mit dem umzugehen, was in seiner Tätigkeit misslingt, und gehorcht nur noch den Befehlen seines Über-Ich.

Dieser positive Gedanke wirkt sich auch auf die Politik des Bundesamts für Gesundheit aus, das diese Entwicklung nur teilweise anerkennt. Im Bereich der Prävention und Gesundheitsförderung hat das BAG begriffen, dass es ohne neue Formen der Interaktion zwischen dem Staat und allen auf diesem Gebiet tätigen Partnern in Zukunft schwieriger sein wird zu handeln. Zwar wurde das Konzept der Prävention und Gesundheitsförderung des BAG um die Einflussfaktoren, Ressourcen und Ungleichheiten bezüglich der Gesundheit erweitert, aber ich hoffe, dass unser politisches Organ auch den Trugbildern des Behaviorismus widerstehen kann. Hier haben unsere Politiker die Chance, ihre Verantwortung wahrzunehmen und denen Schranken zu setzen, die den anderen vorschreiben wollen, was gut für sie ist. Das wünsche ich dem künftigen Präventionsgesetz, das sich zurzeit in Ausarbeitung befindet.

Dr. med. R. Raggenbass Mitglied des Zentralvorstands der FMH Verantwortlicher des Ressorts Gesundheit und Prävention

1 «Nichts zuviel.» Diese von den Lateinern aus dem Griechischen (Mēden agan) entliehene Formel bringt die Haltung zum Ausdruck, dass Übermass und Übertreibung Schaden anrichten.

2 Courier International. 2008;908(27. März):30-5.

3 Layard R. Happiness: Lessons from a new science. London: Penguin Books; 2005. 